\title{
Infarto agudo de miocardio sin lesiones coronarias obstructivas - MINOCA: un enigma para el cardiólogo clínico
}

\author{
Dres. Yamel Ache1*, Carlos Guamán²*, \\ Lorena Viñole ${ }^{1,2}$, Gustavo Vignolo ${ }^{1,2}$
}

\begin{abstract}
Resumen
El infarto agudo de miocardio sin lesiones coronarias obstructivas (MINOCA) es una afección recientemente definida en la práctica cardiológica. Su diagnóstico se basa en la angiografía coronaria, que da inicio a un proceso de búsqueda etiológica muchas veces complejo, pero con importancia pronóstica y terapéutica. Existen diferentes mecanismos involucrados en los MINOCA, que se plantean una vez excluidas la embolia pulmonar, la miocarditis y el síndrome de takotsubo. Se reconoce un patrón epicárdico de MINOCA presente en los casos de disrupción de placa, disección, vasoespasmo, y, eventualmente, embolismo coronario; y un patrón microvascular en casos de espasmo microvascular y embolia coronaria de pequeños vasos. Varias herramientas son útiles en su estudio: ecocardiografía, ventriculografía izquierda, técnicas de imagen intravascular, estudio del flujo coronario y resonancia magnética cardíaca, entre otras. Su uso debe ser guiado por el juicio clínico, y si bien no hay un algoritmo claramente establecido, incorporar todas las herramientas disponibles puede determinar la causa definitiva en algunos pacientes. La presente revisión pretende acercar al cardiólogo clínico información útil para su práctica habitual.
\end{abstract}

Palabras clave: INFARTO DE MIOCARDIO CON ARTERIAS CORONARIAS NO OBSTRUCTIVAS

\section{Acute Myocardial Infarction with Non - Obstructive Coronary Arteries - MINOCA: An enigma for the clinical cardiologist}

\section{Summary}

Acute Myocardial Infarction with Non-Obstructive Coronary Arteries (MINOCA) is a recently defined condition in cardiology practice. The diagnosis starts with coronary angiography that is the beginning of an etiological research process, often complex, but with prognostic and therapeutic importance for each etiology. There are different mechanisms involved in MINOCA, which are posed once pulmonary embolism, myocarditis and takotsubo cardiomyopathy are excluded. It is described an epicardial pattern of MINOCA in cases of plaque disruption, dissection, vasospasm, and eventually coronary embolism; and a microvascular pattern in cases of microvascular spasm and small vessel coronary embolism. Several tools are useful in its study, such as echocardiography, left ventriculography, intravascular imaging techniques, study of coronary flow, and cardiac magnetic resonance, among others. Its use should be guided by clinical criteria. Despite there is no clearly established algorithm in our current practice, it is necessary to incorporate all available tools to approach the definitive MINOCA etiology whenever is possible. The aim of this review is to bring useful information to the clinical cardiologist for his daily practice.

Key words: $\quad$ MYOCARDIAL INFARCTION WITH NON-OBSTRUCTIVE CORONARY ARTERIES

1. Centro Cardiológico Americano, Sanatorio Americano, FEPREMI (Federación de Prestadores Médicos del Interior), Montevideo, Uruguay.

2. Centro Cardiovascular Universitario, Hospital de Clínicas, Facultad de Medicina, Universidad de la República, Montevideo, Uruguay.

* Los autores han contribuido por igual al trabajo y ambos deben ser considerados como primer autor.

Correspondencia: Dra. Yamel Ache. Correo electrónico: yamelache@gmail.com, Dr. Carlos Guamán. Correo electrónico: cgv0792@gmail.com

Los autores declaran no tener conflictos de intereses. 


\section{Infarto Agudo do Miocárdio com Artérias Coronárias Não - Obstrutivas - MINOCA: Um enigma para o cardiologista clínico}

\section{Resumo}

O Infarto Agudo do Miocárdio com Artérias Coronárias Não - Obstrutivas (MINOCA) é uma condição recentemente definida na prática cardiológica. O diagnóstico começa com a angiografia coronariana, que é o início de um processo de pesquisa etiológica, muitas vezes complexo, mas com importância prognóstica e terapêutica para cada etiologia. Existem diferentes mecanismos envolvidos no MINOCA, que são colocados uma vez que a embolia pulmonar, miocardite e cardiomiopatia de takotsubo são excluídos. É descrito um padrão epicárdico de MINOCA presente em casos de ruptura de placa, dissecção, vasoespasmo e embolia coronariana; e um padrão microvascular em casos de espasmo microvascular e embolia coronariana de pequenos vasos. Diversas ferramentas são úteis no estudo de MINOCA: ecocardiografia, ventriculografia esquerda, técnicas de imagem intravascular, estudo do fluxo coronariano, ressonância magnética cardíaca, entre outras. O uso destas deve ser guiado pela suspeita clínica e, embora não haja um processo claramente estabelecido em nossa prática atual, é necessário incorporar todas as ferramentas disponíveis para abordar a etiologia definitiva do MINOCA quando isso for possível. A presente revisão tem como objetivo fornecer ao cardiologista clínico informações úteis para sua prática usual.

Palavras chave: INFARTO DO MIOCÁRDIO COM ARTÉRIAS CORONÁRIAS NÃO OBSTRUTIVAS

\section{Introducción}

La elevación de las troponinas por encima del percentil 99 de la normalidad en un contexto sugerente de isquemia miocárdica, configura la base del diagnóstico clínico de infarto agudo de miocardio $(\mathrm{IAM})^{(1)}$. La etiología más frecuentemente reconocida es la enfermedad coronaria obstructiva (EC)(2). Estudios clásicos demuestran que hasta un $10 \%$ de los pacientes con IAM con elevación del segmento ST no presentan lesiones coronarias obstructivas cuando se estudian en las primeras 24 horas desde el inicio del dolor ${ }^{(3)}$ y que aproximadamente $60 \%$ de los pacientes con IAM sin elevación del segmento ST presentan un vaso ocluido o subocluido en similares condiciones ${ }^{(4)}$. En series actuales la coronariografía no revela lesiones coronarias significativas (obstrucción $\geq 50 \%$ ) en $1 \%$ a $14 \%$ de los $\operatorname{IAM}^{(5-8)}$.

El término MINOCA (infarto agudo de miocardio sin lesiones coronarias obstructivas) fue utilizado por primera vez en el año 2013 por el profesor John Beltrame ${ }^{(9)}$, aunque el paciente que cumple los criterios diagnósticos de IAM, pero sin lesiones coronarias obstructivas, ha representado un dilema etiológico y terapéutico desde el inicio del uso de la angiografía coronaria ${ }^{(10)}$. El interés por esta entidad se ha retomado con mayor impacto desde la aparición de la resonancia magnética cardíaca (RMC) y la imagen intravascular, que han permitido establecer con claridad creciente las diversas causas desencadenantes y desarrollar una estrategia terapéutica $\operatorname{dirigida}^{(7,8,11)}$.

MINOCA se presenta con mayor frecuencia en población femenina y comparativamente más joven, con menos factores de riesgo cardiovascular tradicionales para EC y comorbilidades, pero con mayor frecuencia de estados de hipercoagulabilidad ${ }^{(5,7,8)}$. Se ha descrito una mortalidad hospitalaria en torno a $0,8 \%$, principalmente de causa cardiovascular ${ }^{(12)}$, riesgo de muerte o rehospitalización cardiovascular al año de $11,5 \%{ }^{(12)}$, e incidencia de muerte cardiovascular, IAM o síndrome coronario agudo (SCA), insuficiencia cardíaca y accidente cerebrovascular a los siete años de 17,4\%(13). Por lo tanto, lejos de ser una entidad benigna, MINOCA tiene un pronóstico adverso y puede mostrar un resultado significativamente peor en presencia de $\mathrm{EC}^{(13,14)}$. El conocimiento sobre este tema se ha incrementado de forma exponencial en los últimos años, pero persisten interrogantes que complejizan su abordaje diagnóstico y terapéutico.

\section{Definición y diagnóstico}

Los consensos internacionales más recientes definen como MINOCA a la documentación clínica de IAM junto a la demostración de arterias coronarias sin lesiones obstructivas significativas (tabla 1$)^{(8,15)}$. El problema clínico inicia cuando un paciente con dolor torácico y troponinas positivas o elevación del segmento ST en el electrocardiograma (EGC) presenta una angiografía sin obstrucción coronaria severa. Es aquí donde un análisis cuidadoso y una alta sospecha clínica son fundamentales para descartar enfermedades con alta mortalidad, como disección de aorta o embolia pulmonar y excluir otros diferenciales posibles, a veces con sintomatología poco específica, como miocarditis y miocardiopatía de takotsubo. Una vez alejadas estas etiologías es posible plantear que el paciente presenta MINOCA, iniciando un diagnóstico de trabajo con distintas causas ${ }^{(16)}$.

La identificación etiológica es esencial para definir el tratamiento adecuado a la fisiopatología y al 
Tabla 1. Criterios diagnósticos de MINOCA (modificado de referencia 8).

Criterios diagnósticos de MINOCA

El diagnóstico se realiza después de la angiografía coronaria en un paciente que presenta características clínicas compatibles con un IAM

\section{Criterios de IAM}

(a) Troponina cardíaca positiva definida como un ascenso o descenso de valores seriados, con al menos un valor mayor al percentil 99 de la normalidad.

(b) Evidencia clínica de infarto por al menos uno de los siguientes indicadores:

(i) Síntomas de isquemia.

(ii) Cambios significativos de ST-T nuevos o presumiblemente nuevos, o BRI nuevo.

(iii) Desarrollo de ondas Q patológicas.

(iv) Pruebas de imagen con nueva pérdida de miocardio viable.

(v) Trombo intracoronario evidente en la angiografía o en la autopsia.

Arterias coronarias no obstructivas en la angiografía

Ausencia de enfermedad coronaria obstructiva en la angiografía (es decir, sin estenosis coronaria $\geq 50 \%$ ), en cualquier posible arteria relacionada con el infarto.

Esto incluye a pacientes con:

- Arterias coronarias normales (estenosis < 30\%).

- Ateromatosis coronaria leve (estenosis $>30 \%$ pero $<50 \%)$.

Ausencia de causa clínica específica para la presentación aguda

En el momento de la angiografía el diagnóstico específico no es aparente. Existe, por lo tanto, la necesidad de seguir evaluando al paciente por la causa subyacente al MINOCA.

pronóstico del paciente ${ }^{(13,15-17)}$. Las herramientas diagnósticas iniciales del MINOCA incluyen la historia clínica, ECG, troponinas, ecocardiografía transtorácica, angiografía coronaria con test de vasorreactividad y ventriculografía izquierda ${ }^{(18)}$. El motivo de consulta suele ser dolor torácico y se manifiesta en el ECG más frecuentemente sin elevación del segmento $\mathrm{ST}^{(5,8)}$. El valor de corte angiográfico de obstrucción coronaria $<50 \%$ es arbitrario y deben reconocerse factores limitantes: existe variabilidad inter e intraobservador de la estimación angiográfica de las estenosis, la trombosis es un proceso dinámico y la presencia de estenosis coronarias significativas no descarta de forma absoluta la posibilidad de MINOCA ${ }^{(8,19)}$.

En el escenario agudo se recomienda realizar ventriculografía izquierda o un ecocardiograma transtorácico, o ambos, para valorar la existencia de alteraciones segmentarias de la contractilidad. La embolia pulmonar puede ser la causa de la injuria miocárdica y la aproximación diagnóstica será mediante dímeros D o angiotomografía, o ambos, de arterias pulmonares ${ }^{(8,14,18)}$. Se debe considerar el uso de RMC con realce tardío con gadolinio, imagen intracoronaria con ultrasonido intravascular (IVUS), o con tomografía de coherencia óptica (OCT) $)^{(8,14,16,18)}$. No hay un algoritmo consensuado para el abordaje de los MINOCA, pero diferentes estrategias han sido propuestas en artículos de opinión y de posición, y pueden ayudar a la toma de decisiones (figura $1)^{(8,14) \text {. }}$

\section{Causas de MINOCA}

Basándose en el resultado de la ventriculografía izquierda se reconoce la existencia de dos patrones característicos ${ }^{(18)}$ (figura 2):

- Patrón epicárdico: presenta anomalías de la motilidad en el territorio de una arteria coronaria epicárdica.

- Patrón microvascular: las anomalías de la motilidad son regionales, con una extensión mayor a la determinada por una única arteria coronaria epicárdica.

\section{Patrón epicárdico}

\section{Disrupción de placa}

La disrupción de placa, principal etiología del IAM tipo 1, puede presentarse sin EC significativa y en ausencia de trombo intracoronario, aunque los pacientes suelen tener algún grado de aterosclerosis, así sea mínimo(8,18). La ausencia de estenosis coronarias significativas en la angiografía no excluye la posibilidad de accidente de placa y los estudios muestran que las técnicas de imagen intravascular permiten identificar rotura, ulceración o erosión de placa hasta en $40 \%$ de los pacientes con MINO$\mathrm{CA}^{(20,21)}$. Dadas las implicancias de este hallazgo, tanto el IVUS como la OCT deberían formar parte del algoritmo diagnóstico, siendo la OCT la técnica preferida por su mayor resolución ${ }^{(8,15,16,18)}$.

Es conocido que la trombosis es un fenómeno dinámico, donde la lisis endógena existe como mecanismo protector frente a la obstrucción coronaria, lo que explica la ausencia de lesión coronaria obstructiva en presencia de una disrupción de placa aterosclerótica $^{(22)}$. La necrosis resulta de la interac- 


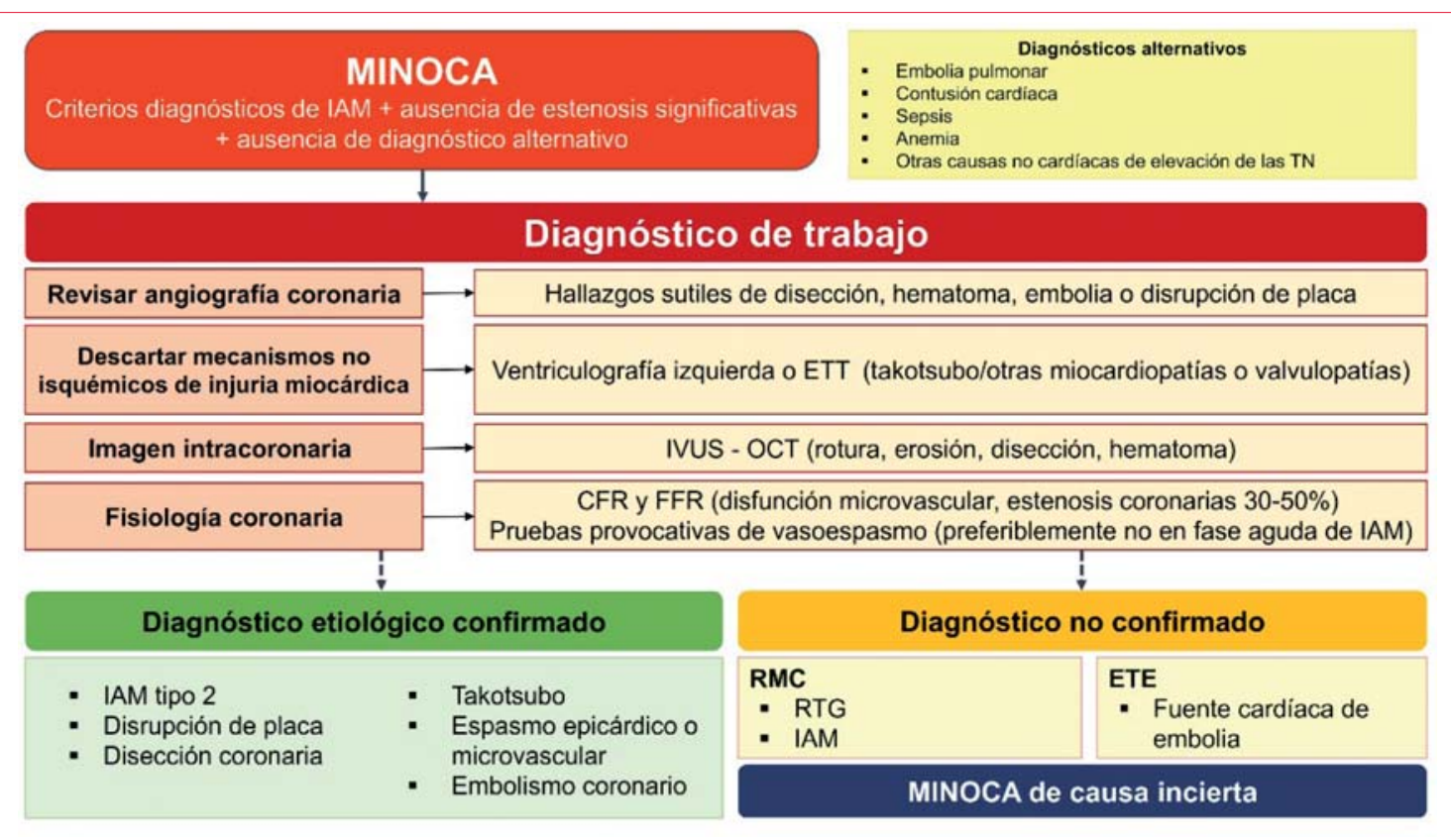

Figura 1. Algoritmo diagnóstico en MINOCA (modificado de referencias 8 y 15). TN: troponinas; ETT: ecocardiograma transtorácico; CFR: reserva de flujo coronario; FFR: reserva fraccional de flujo; RTG: realce tardío con gadolinio; ETE: ecocardiograma transesofágico.

ción entre la trombosis, el embolismo y el vasoespasmo que se suceden antes y durante la lisis del trombo y puede ser evidenciada mediante RMC. Esta suele mostrar edema miocárdico en áreas grandes, y, eventualmente, áreas de necrosis más pequeñas en el territorio vascular involucrado, hallazgos que apoyan este mecanismo, aunque no descartan vasoespasmo coronario epicárdico como factor etiológico aislado. En otros casos, la RMC puede mostrar una pequeña área bien definida de realce tardío con gadolinio, posiblemente secundaria a obstrucción de vasos pequeños, lo que sugiere embolización de restos aterotrombóticos ${ }^{(8,16,18)}$. La terapia antiplaquetaria recomendada en el IAM y las estatinas están indicadas en los pacientes con sospecha o confirmación de disrupción de placa y $\operatorname{MINOCA}^{(16,23)}$.

\section{Disección coronaria espontánea}

Es la etiología responsable de hasta una cuarta parte de los SCA en mujeres menores de 50 años ${ }^{(24)}$, y de $1,7 \%$ a $4 \%$ de los SCA en general ${ }^{(25-28)}$. Mucho más frecuente en mujeres con escasos factores de riesgo cardiovascular, representa la principal causa de SCA durante el embarazo y parto ${ }^{(28)}$. Genera obstrucción de la luz coronaria con la consecuente isquemia miocárdica y puede no ser evidente en la angiografía coronaria, como puede ocurrir con el hematoma intramural, por lo que la imagen intracoronaria muchas veces es esencial en el diagnóstico ${ }^{(25)}$. La arteria descendente anterior es la más frecuentemente involucrada ${ }^{(28)}$.
Suele manifestarse electrocardiográficamente con elevación del segmento ST y troponinas muy elevadas. Varios factores han sido implicados en la generación de la disección, como la presencia de displasia fibromuscular en la vasculatura coronaria, factores genéticos, influencia hormonal, enfermedades inflamatorias sistémicas, junto a desencadenantes ambientales o estresores ${ }^{(16,18,25,28)}$.

Se recomienda un manejo conservador en la mayoría de los casos, y los estudios demuestran que la disección suele resolverse espontáneamente luego de los primeros 35 días ${ }^{(25,28,29)}$. En los pacientes con disección de tronco de la coronaria izquier$\mathrm{da}$, isquemia persistente o inestabilidad hemodinámica, puede ser necesario recurrir a la revascularización mediante angioplastia o cirugía de revascularización miocárdica, según las características de la disección y la experiencia del centro ${ }^{(25,28)}$. La angioplastia de estas lesiones puede generar progresión de la disección, por lo que no se recomienda su uso sistemático ${ }^{(25)}$. Si bien no existe evidencia sólida al respecto, hay consensos que recomiendan el uso de terapia antiplaquetaria doble, y, recientemente, la terapia antiplaquetaria única ${ }^{(15)}$ y betabloqueantes, sin estar indicada la terapia con estatinas dado que generalmente ocurre en ausencia de enfermedad aterosclerótica ${ }^{(15,16,25,28)}$. El pronóstico se encuentra signado por una tasa de eventos adversos a mediano y largo plazo como dolor torácico, ansiedad y depresión, eventos cardíacos mayores, IAM recurrente, disección recurren- 


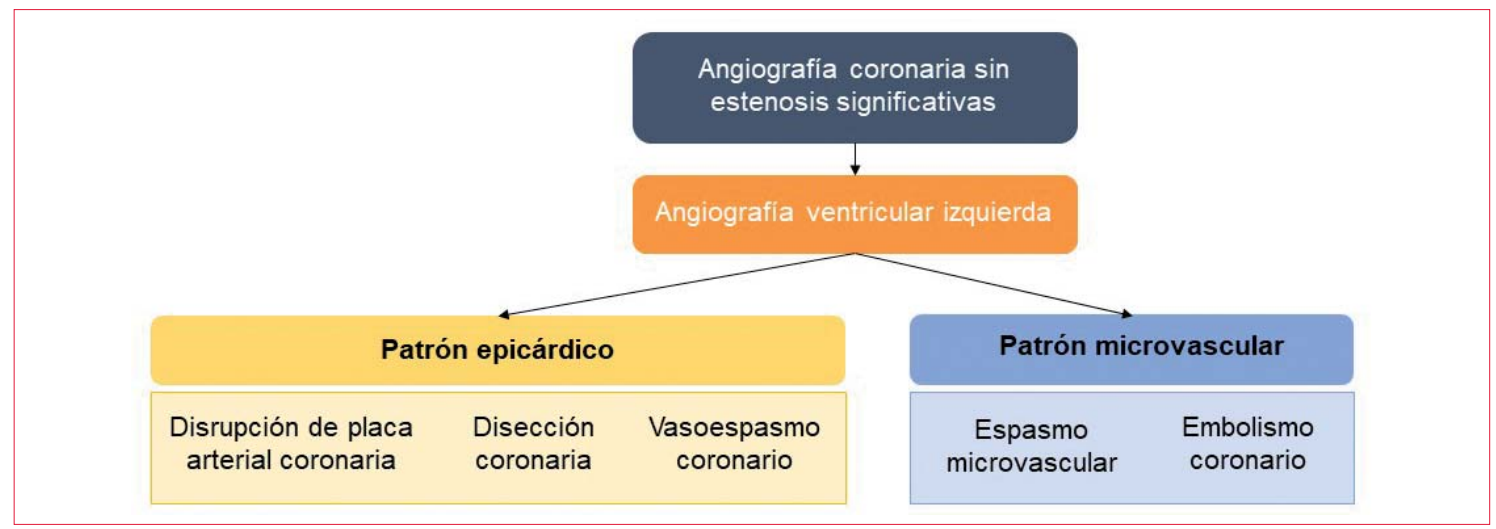

Figura 2. Causas de MINOCA (modificado de referencia 18).

te, revascularización anticipada, insuficiencia cardíaca congestiva, accidente cerebrovascular y muerte, de entre $10 \%$ y $30 \%$ a los tres años, siendo el más frecuente de estos eventos la recurrencia de la disección ${ }^{(28)}$.

\section{Vasoespasmo coronario}

Las pruebas provocadoras de espasmo han resultado positivas en $27 \%$ a $30 \%$ de pacientes con MINO$\mathrm{CA}^{(8,30)}$.

En la fisiopatología del vasoespasmo está implicada la reactividad aumentada del músculo liso a sustancias endógenas o exógenas, como el tabaco, fármacos (triptanos, pseudoefedrina, inotrópicos), drogas de uso recreativo (metanfetaminas, cocaína), hipertensión y estrés ${ }^{(18)}$. Clínicamente puede manifestarse como angina crónica atípica o como un evento agudo intercurrente ${ }^{(8)}$. El paro cardíaco y la muerte súbita son un riesgo en estos pacientes, y pueden ocurrir en ausencia de $\mathrm{EC}^{(14)}$. Son sugestivos de vasoespasmo los episodios recurrentes de dolor en reposo, que responden rápidamente a nitratos de corta duración, especialmente si está acompañado de cambios transitorios del segmento ST en el ECG y de un patrón circadiano nocturno ${ }^{(8)}$. Por tratarse de un fenómeno dinámico su diagnóstico es muy difícil sin un test de provocación. Las pruebas de provocación durante la angiografía coronaria con ergonovina o acetilcolina intracoronarias han demostrado ser efectivas en el diagnóstico, siendo preferida la última por su corta vida media. El resultado positivo se asocia a un pronóstico clínico adverso, en cuanto a mortalidad cardiovascular, mortalidad por cualquier causa, SCA recurrente y peor calidad de vida ${ }^{(31)}$. No obstante, algunos autores recomiendan no realizarlas por el riesgo de vasoespasmo intratable, fundamentalmente vinculado al uso de ergonovina ${ }^{(14)}$. Por este motivo, se recomienda diferir los estudios de vasorreactividad con respecto al escenario agudo ${ }^{(8)}$. Montone y colaboradores repor- taron los primeros datos de seguridad de una prueba provocadora de espasmo (usando ergonovina o acetilcolina) en las primeras 48 horas de admisión en 80 pacientes con MINOCA, que fue positiva en 37 pacientes $(46,2 \%)$ y no registraron eventos adversos mayores, incluyendo infarto recurrente o muerte ${ }^{(31)}$.

Los calcioantagonistas son el tratamiento de elección; en casos de angina refractaria pueden asociarse dihidropirídinicos y no dihidropiridínicos. Los nitratos son de utilidad en la fase aguda, pero su beneficio a largo plazo es menos claro. Otros fármacos que han demostrado ser efectivos son nicorandil y cilostazol $8,15,18,30)$. En pacientes con eventos arrítmicos de riesgo para muerte súbita, puede ser necesario considerar el implante de un cardiodesfibrilador ${ }^{(8,32)}$.

\section{Patrón microvascular}

\section{Espasmo microvascular}

De acuerdo con los estudios disponibles, el 25\% de los MINOCA tiene evidencia de espasmo microvascular, y algunos autores consideran este cuadro clínico como la expresión inestable de la angina microvascular crónica, siendo difícil establecer la superposición entre ambas.

La disfunción microvascular puede ser tanto la causa de la isquemia como una secuela de la injuria miocárdica isquémica o no isquémica ${ }^{(15,33)}$. Es más común en mujeres y en pacientes con factores de riesgo cardiovascular. La isquemia miocárdica se hace evidente a través del dolor anginoso acompañado de cambios electrocardiográficos, como desviación del segmento ST o la presencia de ondas T acuminadas en al menos dos derivaciones contiguas $^{(18,33)}$.

Se ha utilizado acetilcolina intracoronaria, nitroprusiato de sodio, nitroglicerina y adenosina para valorar la disfunción microvascular, y el resultado patológico se relaciona en forma independiente 
con eventos cardiovasculares agudos en pacientes con y sin $\mathrm{EC}^{(14)}$. Pueden ser de utilidad las pruebas de reserva de flujo coronario para el estudio de la enfermedad de la microvasculatura ${ }^{(8,14)}$. El diagnóstico de disfunción de flujo coronario puede ser determinado por cualquiera de los siguientes criterios: 1 ) reserva de flujo coronario $<2,0$ en respuesta a un estímulo vasodilatador como adenosina; 2 ) evidencia de espasmo microvascular diagnosticado durante prueba provocadora de espasmo: dolor torácico y cambios sugestivos de isquemia en el ECG inducidos por provocación de acetilcolina en ausencia de espasmo epicárdico (reducción del diámetro > $75 \%)$, o 3) flujo coronario deteriorado medido con recuento de cuadros TIMI (Thrombolysis in Myocardial Infarction) corregida ${ }^{(15,34,35)}$.

La angina por vasoespasmo microvascular persiste a pesar del tratamiento vasodilatador con antagonistas de los canales de calcio hasta en un 35\% de los pacientes, con repercusión en la calidad de vi$\mathrm{da}^{(35)}$, y es un posible sustrato fisiopatológico y anatómico para el desarrollo de insuficiencia cardía$\mathrm{ca}^{(36)}$. Los fármacos que han demostrado mayor beneficio en el alivio de la sintomatología son los calcioantagonistas y betabloqueantes, aunque no existen ensayos clínicos sobre este tema ${ }^{(15)}$.

\section{Embolismo coronario}

El embolismo coronario se incluye dentro del patrón microvascular en la clasificación propuesta, pero el émbolo puede ocluir una arteria coronaria de mayor calibre y presentarse como patrón epicárdico de MINOCA, o como una lesión coronaria obstructiva $^{(18)}$. Puede originarse a partir de fuentes trombóticas cardíacas, como fibrilación auricular, valvulopatías, trombos intracavitarios, o a partir de trombos sistémicos, destacándose la presencia de desórdenes trombóticos hereditarios (factor V Leiden, deficiencia de proteínas $\mathrm{C}$ o S) o adquiridos (síndrome antifosfolipídico, trastornos mieloproliferativos) $)^{(14,18)}$.

Estudios de tamizaje de trombofilia en pacientes con MINOCA han reportado una prevalencia de $14 \%$ de desórdenes hereditarios ${ }^{(8)}$. La embolia no trombótica es ocasionalmente causa de MINOCA, ya sea a partir de vegetaciones valvulares en endocarditis bacteriana y no bacteriana, tumores cardíacos como mixomas y fibroelastoma papilar, embolias de aire o calcio ${ }^{(14,18)}$.

El embolismo coronario es responsable de hasta $4,3 \%$ de los casos de IAM con elevación del segmento ST en series recientes, y se asocia a una mayor mortalidad respecto a SCA de otras etiologías ${ }^{(37)}$. La embolia paradójica, que puede estar relacionada con foramen oval permeable, defecto septal atrial grande o fístula arteriovenosa coronaria ${ }^{(18)}$, se ha encontrado en $0,45 \%$ de los pacientes de una serie de 4.848 personas con IAM, estimándose una incidencia de al menos 10 casos por millón ${ }^{(38)}$. Existe un importante subdiagnóstico de la embolia coronaria en los pacientes con MINOCA, al igual que sucede con el resto de las etiologías. El tratamiento dependerá de la causa subyacente a la embolia( ${ }^{(18)}$. La anticoagulación oral por tres meses estaría recomendada si la causa es un factor de riesgo procoagulante temporal y a largo plazo en presencia de un factor de riesgo persistente, como fibrilación auricular con score $\mathrm{CHA}_{2} \mathrm{DS}_{2}-\mathrm{VASc} \geq 2^{(8,18)}$.

\section{Síndrome de takotsubo}

Habitualmente desencadenado por un estrés emocional o físico, el síndrome de takotsubo suele presentarse como un SCA con o sin elevación del segmento ST, y es generador de una disfunción ventricular característicamente transitoria, de gravedad variable. En las últimas revisiones se recomienda descartar la presencia de takotsubo antes de plantear el diagnóstico de MINOCA ${ }^{(1,15)}$.

Los criterios diagnósticos internacionales propuestos recientemente (InterTAK Diagnostic Criteria) incluyen ${ }^{(39,40)}$ :

1. Hipoquinesia, aquinesia o disquinesia transitoria de segmentos medios, basales o focales del ventrículo izquierdo, incluyendo el balonamiento apical. Las anomalías del movimiento son regionales y exceden una distribución vascular epicárdica, pero no es excluyente dado que puede existir un patrón focal en un territorio coronario.

2. El desencadenante suele preceder a la presentación clínica, pero puede no estar presente.

3. Los trastornos neurológicos como la hemorragia subaracnoidea, accidente cerebrovascular, convulsiones y el feocromocitoma pueden ser desencadenantes.

4. Suele presentar alteraciones en el ECG como elevación o depresión del segmento ST, inversión de la onda $\mathrm{T}$ y prolongación del intervalo QT corregido, pero existen casos infrecuentes, sin ningún cambio en el ECG.

5. Los biomarcadores cardíacos están moderadamente elevados en la mayoría de los casos. Es frecuente la elevación significativa del péptido natriurético cerebral.

6. La presencia de EC significativa no excluye la presencia de takotsubo.

7. Afecta generalmente a mujeres posmenopáusicas.

8. Ausencia de miocarditis. 
La fisiopatología es compleja y variable, la estimulación simpática tiene un rol central con el consecuente aumento de las catecolaminas ${ }^{(18,39)}$, que puede ser causante de aturdimiento miocárdico por cardiotoxicidad, de espasmo epicárdico de múltiples vasos o espasmo microvascular, e incluso de accidente de placa y lisis espontánea de un trombo coronario $^{(18,39)}$. Puede presentarse con un amplio espectro clínico, desde una afectación muy leve y difícil de diagnosticar, hasta cuadros de shock cardiogénico y muerte ${ }^{(39)}$. La ventriculografía izquierda es la herramienta diagnóstica de elección ${ }^{(8,18,39)}$. La ecocardiografía y la RMC son también valiosas en el proceso diagnóstico, siendo fundamental la última para excluir etiologías diferenciales de $\operatorname{MINOCA}^{(40)}$.

\section{Miocarditis}

En pacientes jóvenes los síntomas de miocarditis aguda pueden emular a los del IAM. Assomull y colaboradores ${ }^{(11)}$ analizaron pacientes con dolor torácico, troponinas positivas y angiografía sin lesiones coronarias obstructivas, encontrando signos de miocarditis en 50\% de las RMC. En una revisión sistemática, la miocarditis fue la causa de MINOCA en $33 \%$ de los pacientes ${ }^{(7)}$. Recientemente, la cuarta definición de IAM estableció el concepto de injuria miocárdica como la elevación de las troponinas cardíacas de causa no isquémica, por lo que actualmente la miocarditis no es considerada etiología de MINOCA al no cumplir sus criterios diagnósti$\cos ^{(1,15)}$. Su presentación clínica es variable, en pacientes con un cuadro clínico clásico, el diagnóstico puede realizarse antes de la angiografía coronaria, pero en muchos escenarios efectuar el diagnóstico no es fácil y depende de una alta sospecha clínica.

Dada su importancia pronóstica y su manejo diferente respecto al IAM, la miocarditis debe ser considerada antes de iniciar la elaboración diagnóstica de $\operatorname{MINOCA}^{(8,9,15)}$.

\section{Tromboembolia pulmonar}

La tromboembolia pulmonar puede ser causa de elevación de las troponinas cardíacas. Al ser una patología de alta mortalidad y con tratamiento efectivo conocido, hay que sospecharla antes de efectuar el diagnóstico de MINOCA ${ }^{(8,15,41)}$. La angiotomografía de arterias pulmonares debe ser solicitada con fundamento clínico y no está indicada en todos los pacientes sin lesiones coronarias obstructivas ${ }^{(9,41)}$.

\section{Tratamiento}

En los pacientes con MINOCA, el tratamiento no es la revascularización coronaria, lo que conlleva una difi- cultad terapéutica al no existir guías estandarizadas para su abordaje. Un análisis por puntaje de propensión de 9.136 pacientes con MINOCA del registro SWEDEHEART, comprobó que el tratamiento con estatinas, con agentes moduladores del sistema renina-angiotensina, y posiblemente con betabloqueantes, disminuye el riesgo de eventos adversos cardiovasculares mayores, no así el tratamiento con agentes $\operatorname{antiplaquetarios}^{(17)}$. Sin embargo, hasta la fecha no existen ensayos clínicos randomizados sobre tratamiento del MINOCA y dada la heterogeneidad de los pacientes incluidos bajo este término tan amplio, el consenso actual es que debe realizarse un abordaje individualizado según el mecanismo fisiopatológico involucrado (tabla 2$)^{(11,13,15)}$.

\section{Pronóstico}

Ciliberti y colaboradores reportaron, tras el análisis de un registro retrospectivo, una mortalidad de $17,4 \%$ en el seguimiento a siete años en pacientes con $\mathrm{MINOCA}^{(13)}$. De acuerdo con los datos de un registro canadiense que analizó 2.092 pacientes afectados por MINOCA, la mortalidad hospitalaria fue de $0,8 \%$ versus $2,7 \%$ observada en pacientes con SCA y EC. La tasa de muerte o reinfarto al año fue significativamente mayor en los pacientes con EC, pero se registró un valor de 5,3\% en los pacientes con $\mathrm{MINOCA}^{(12)}$. Por lo tanto, la información disponible desestima su benignidad, aunque presentan un pronóstico más favorable que los pacientes con EC. No existen estudios prospectivos publicados que aporten datos definiti$\operatorname{vos}^{(14,42)}$. La concomitancia de EC ensombrece el pronóstico de los MINOCA, incluso cuando las estenosis coronarias son leves a moderadas ${ }^{(13,14)}$, pero su ausencia no implica buen pronóstico(43).

El número de arterias coronarias enfermas, independientemente de la severidad, se ha relacionado con peores resultados a largo plazo ${ }^{(13)}$. Otros factores de mal pronóstico son: la edad, tabaquismo, diabetes, hipertensión arterial, fracción de eyección ventricular izquierda reducida y presencia de enfermedad pulmonar obstructiva crónica, de manera similar a lo que ocurre en pacientes con EC $^{(43,44)}$. Las mujeres tienen mayor incidencia de eventos cardiovasculares adversos en el seguimiento ${ }^{(45)}$.

\section{Conclusiones}

El MINOCA requiere una elaboración diagnóstica con un amplio abanico de etiologías a sospechar de acuerdo con el contexto clínico. Esto posibilita la aproximación a patologías con diferente valor pronóstico que se benefician de tratamientos específi- 
Tabla 2. Principales mecanismos del MINOCA con su potencial tratamiento (modificado de referencia 16).

\begin{tabular}{|c|c|c|}
\hline Mecanismo fisiopatológico & Investigación clínica & Terapia potencial \\
\hline $\begin{array}{l}\text { Disrupción de placa: } \\
\text { - Erosión de placa } \\
\text { - Ruptura de placa }\end{array}$ & $\begin{array}{l}\text { - Tomografía de coherencia óptica } \\
\text { - Ultrasonido intravascular }\end{array}$ & - Estatinas \\
\hline $\begin{array}{l}\text { Tromboembolismo coronario: } \\
\text { - Trombosis } \\
\text { - Embolismo }\end{array}$ & $\begin{array}{l}\text { - Tomografía de coherencia óptica } \\
\text { - Trombofilia congénita } \\
\text { - Trombofilia adquirida }\end{array}$ & $\begin{array}{l}\text { - Agentes antiplaquetarios } \\
\text { - Anticoagulantes }\end{array}$ \\
\hline $\begin{array}{l}\text { Disfunción vasomotora } \\
\text { coronaria: } \\
\text { - Espasmo coronario } \\
\text {. Disfunción microvascular }\end{array}$ & $\begin{array}{l}\text { - Test de espasmo coronario } \\
\text { - Reserva de flujo fraccional } \\
\text { - Índices de resistencia } \\
\text { microvascular }\end{array}$ & $\begin{array}{l}\text { - Bloqueadores de los canales de } \\
\text { calcio }\end{array}$ \\
\hline
\end{tabular}

cos no incluidos en las recomendaciones para los IAM de acuerdo con la práctica clínica actual.

\section{Perspectivas}

La terminología que engloba al MINOCA es motivo de discusión, de manera que hay investigadores que lo incluyen dentro de un grupo más abarcativo denominado Síndromes Coronarios Agudos en pacientes con Arterias Coronarias Angiográficamente Normales o Casi Normales (ACS-NNOCA), integrando además a los pacientes que se presentan con angina inestable ${ }^{(14,19)}$. A pesar de los acuerdos, referidos fundamentalmente al uso de la ventriculografía izquierda, imagen intracoronaria, ecocardiografía y RMC, permanecen sin respuesta interrogantes importantes, como la pertinencia y oportunidad de pruebas de vasorreactividad coronaria(14,31). En nuestro medio, donde los recursos diagnósticos son limitados, muchas veces no se logra conocer la etiología involucrada, permaneciendo estos pacientes dentro de la categoría de causa incierta. La cardiología ha avanzado enormemente en el tratamiento de la EC, pero este grupo de pacientes sin lesiones coronarias obstructivas ha sido rezagado durante años al creer que su pronóstico no llegaría a ser fatal o que eran consecuencia de troponinas falsamente positivas $^{(9)}$. En Latinoamérica, no existe información publicada sobre la evolución de este grupo de pacientes y si son sometidos al procedimiento diagnóstico recomendado. Parecería, por lo tanto, importante aunar esfuerzos y diseñar un registro nacional con el objetivo de progresar en el abordaje integral del IAM.

Yamel Ache, https://orcid.org/0000-0001-9956-4081 Carlos Guamán, https://orcid.org/0000-0002-1065-1988 Lorena Viñole, https://orcid.org/0000-0003-0087-7948 Gustavo Vignolo, https://orcid.org/0000-0002-4851-5316

\section{Bibliografía}

1. Thygesen K, Alpert J, Jaffe A, Chaitman B, Bax J, Morrow D, et al. Fourth universal definition of myocardial infarction (2018). Eur Heart J. 2019; 40(3):237-69. doi: 10.1093/eurheartj/ehy462

2. Ibanez B, James S, Agewall S, Antunes M, Bucciarelli-Ducci C, Bueno H, et al. 2017 ESC Guidelines for the management of acute myocardial infarction in patients presenting with ST-segment elevation: The Task Force for the management of acute myocardial infarction in patients presenting with ST-segment elevation of the European Society of Cardiology (ESC). Eur Heart J. 2018; 39(2):119-177. doi: 10.1093/eurheartj/ehx393

3. DeWood M, Spores J, Notske R, Mouser L, Burroughs R, Golden M, et al. Prevalence of total coronary occlusion during the early hours of transmural myocardial infarction. N Engl J Med. 1980; 303(16): 897-902. doi: 10.1056/NEJM198010163031601

4. DeWood M, Stifter W, Simpson C, Spores J, Eugster G, Judge T, et al. Coronary arteriographic findings soon after non-q-wave myocardial infarction. N Engl J Med. 1986; 315(7):417-23. doi: 10.1056/ NEJM198608143150703

5. Safdar B, Spatz E, Dreyer R, Beltrame J, Lichtman J, Spertus J, et al. Presentation, clinical profile, and prognosis of young patients with myocardial infarction with nonobstructive coronary arteries (MINOCA): results from the VIRGO study. J Am Heart Assoc. 2018; 7(13):e009174. doi: 10.1161/ JAHA.118.009174

6. von Korn H, Graefe V, Ohlow M, Yu J, Huegl B, Wagner A, et al. Acute coronary syndrome without significant stenosis on angiography: characteristics and prognosis. Tex Heart Inst J. 2008; 35(4):406-12.

7. Pasupathy S, Air T, Dreyer R, Tavella R, Beltrame J. Systematic review of patients presenting with suspected myocardial infarction and nonobstructive coronary arteries. Circulation 2015; 131(10):861-70. doi: 10.1161/CIRCULATIONAHA.114.011201 
8. Agewall S, Beltrame J, Reynolds H, Niessner A, Rosano G, Caforio A, et al. ESC working group position paper on myocardial infarction with non-obstructive coronary arteries. Eur Heart J. 2017; 38(3):143-53. doi: 10.1093/eurheartj/ehw149

9. Beltrame J. Assessing patients with myocardial infarction and nonobstructed coronary arteries (MINOCA). J Intern Med. 2013; 273(2):182-5. doi: 10.1111/j.1365-2796.2012.02591.x

10. Larsen A, Galbraith P, Ghali W, Norris C, Graham M, Knudtson M, et al. Characteristics and outcomes of patients with acute myocardial infarction and angiographically normal coronary arteries. Am J Cardiol. 2005; 95(2):261-3. doi: 10.1016/ j.amjcard.2004.09.014

11. Assomull R, Lyne J, Keenan N, Gulati A, Bunce $\mathbf{N}$, Davies S, et al. The role of cardiovascular magnetic resonance in patients presenting with chest pain, raised troponin, and unobstructed coronary arteries. Eur Heart J. 2007; 28(10):1242-9. doi: 10 1093/eurheartj/ehm113

12. Bainey K, Welsh R, Alemayehu W, Westerhout C, Traboulsi D, Anderson T, et al. Population-level incidence and outcomes of myocardial infarction with non-obstructive coronary arteries (MINOCA): Insights from the Alberta contemporary acute coronary syndrome patients invasive treatment strategies (COAPT) study. Int J Cardiol. 2018; 264:12-17. doi: 10.1016/j.ijcard.2018.04.004

13. Ciliberti G, Coiro S, Tritto I, Benedetti M, Guerra F, Del Pinto M, et al. Predictors of poor clinical outcomes in patients with acute myocardial infarction and non-obstructed coronary arteries (MINOCA). Int J Cardiol. 2018; 267:41-45. doi: 10. 1016/j.ijcard.2018.03.092

14. Manolis A, Manolis A, Manolis T, Melita H. Acute coronary syndromes in patients with angiographically normal or near normal (non-obstructive) coronary arteries. Trends Cardiovasc Med. 2018; 28(8):541-551. doi: 10.1016/j.tcm.2018.05.006

15. Tamis-Holland J, Jneid H, Reynolds H, Agewall S, Brilakis E, Brown T, et al. Contemporary diagnosis and management of patients with myocardial infarction in the absence of obstructive coronary artery disease: a scientific statement from the American Heart Association. Circulation 2019; 139(18):e891-908. doi: 10. 1161/CIR.0000000000000670

16. Tavella R, Pasupathy S, Beltrame J. MINOCA: a personalised medicine approach. Int J Cardiol. 2018; 267:54-55. doi: 10.1016/j.ijcard.2018.05.077

17. Lindahl B, Baron T, Erlinge D, Hadziosmanovic N, Nordenskjöld A, Gard A, et al. Medical Therapy for secondary prevention and long-term outcome in patients with myocardial infarction with nonobstructive coronary artery disease. Circulation 2017;135(16) 1481-9. doi: 10.1161/CIRCULATIONAHA. 116.026336
18. Scalone G, Niccoli G, Crea F. Editor's Choice: pathophysiology, diagnosis and management of MINOCA: an update. Eur Heart J Acute Cardiovasc Care 2019; 8(1):54-62. doi: $10.1177 / 2048872618782414$.

19. Lyubarova R, Schulman-Marcus J. Editorial commentary: naming the misfits - MINOCA, ACSNNOCA, and the rest of the family. Trends Cardiovasc Med. 2018;2 8(8):552-553. doi: 10.1016/ j.tcm.2018.05.012

20. Reynolds H, Srichai M, Iqbal S, Slater J, Mancini G, Feit F, et al. Mechanisms of myocardial infarction in women without angiographically obstructive coronary artery disease. Circulation 2011; 124(13):1414-25. doi: 10.1161/CIRCULATIONAHA.111.026542

21. Ouldzein H, Elbaz M, Roncalli J, Cagnac R, Carrié D, Puel J, et al. Plaque rupture and morphological characteristics of the culprit lesion in acute coronary syndromes without significant angiographic lesion: analysis by intravascular ultrasound. Ann Cardiol Angeiol. (Paris) 2012; 61(1):20-6. doi: 10.1016/j.ancard.2011.07.011

22. Kovacs I, Gorog D, Yamamoto J. Enhanced spontaneous thrombolysis: a new therapeutic challenge. $\mathrm{J}$ Thromb Thrombolysis 2006; 21(3):221-7. doi: 10.1007/s11239-006-6579-0

23. Toth G, Toth B, Johnson N, De Vroey F, Di Serafino L, Pyxaras S, et al. Revascularization decisions in patients with stable angina and intermediate lesions: results of the international survey on interventional strategy. Circ Cardiovasc Interv. 2014; 7(6):751-9. doi: 10.1161/CIRCINTERVENTIONS.114.001608

24. Saw J, Aymong E, Mancini G, Sedlak T, Starovoytov $A$, Ricci $D$. Nonatherosclerotic coronary artery disease in young women. Can J Cardiol. 2014; 30(7):814-9. doi: 10.1016/j.cjca.2014.01.011

25. Saw J, Mancini G, Humphries K. Contemporary review on spontaneous coronary artery dissection. J Am Coll Cardiol 2016; 68(3):297-312. doi: 10.1016/j.jacc.2016.05.034. Erratum in: J Am Coll Cardiol. 2016; 68(14):1606.

26. Rashid H, Wong $\mathbf{D}$, Wijesekera $\mathbf{H}$, Gutman $\mathbf{S}$, Shanmugam V, Gulati R, et al. Incidence and characterisation of spontaneous coronary artery dissection as a cause of acute coronary syndrome-A single-centre Australian experience. Int J Cardiol. 2016; 202:336-8. doi: 10.1016/j.ijcard.2015.09.072

27. Nishiguchi T, Tanaka A, Ozaki Y, Taruya A, Fukuda S, Taguchi H, et al. Prevalence of spontaneous coronary artery dissection in patients with acute coronary syndrome. Eur Heart J Acute Cardiovasc Care 2016; 5(3):263-70. doi: 10.1177/ 2048872613504310

28. Hayes S, Kim E, Saw J, Adlam D, Arslanian-Engoren $\mathbf{C}$, Economy $\mathbf{K}$, et al. Spontaneous coronary artery dissection: current state of the science: a scientific statement from the American Heart 
Association. Circulation 2018; 137(19):e523-57. doi 10.1161/CIR.0000000000000564

29. Tweet M, Eleid M, Best P, Lennon R, Lerman A, Rihal C, et al. Spontaneous coronary artery dissection: revascularization versus conservative therapy. Circ Cardiovasc Interv. 2014; 7(6):777-86. doi: 10.1161/CIRCINTERVENTIONS.114.001659

30. Ong P, Athanasiadis A, Hill S, Vogelsberg H, Voehringer M, Sechtem U. Coronary artery spasm as a frequent cause of acute coronary syndrome: The CASPAR (Coronary Artery Spasm in Patients With Acute Coronary Syndrome) study. J Am Coll Cardiol. 2008; 52(7):523-7. doi: 10.1016/ j.jacc.2008.04.050

31. Montone R, Niccoli G, Fracassi F, Russo M, Gurgoglione F, Cammà G, et al. Patients with acute myocardial infarction and non-obstructive coronary arteries: safety and prognostic relevance of invasive coronary provocative tests. Eur Heart J. 2018; 39(2):91-98. doi: 10.1093/eurheartj/ehx667

32. Lanza G, Sestito A, Sgueglia G, Infusino F, Manolfi M, Crea F, et al. Current clinical features, diagnostic assessment and prognostic determinants of patients with variant angina. Int J Cardiol. 2007; 118(1):41-7. doi: 10.1016/j.ijcard.2006.06.016

33. Lanza G, Crea F. Primary coronary microvascular dysfunction: clinical presentation, pathophysiology, and management. Circulation 2010; 121(21):2317-25. doi: 10.1161/CIRCULATIONAHA.109.900191

34. Ong P, Camici P, Beltrame J, Crea F, Shimokawa $\mathrm{H}$, Sechtem U, et al. International standardization of diagnostic criteria for microvascular angina. Int J Cardiol. 2018; 250:16-20. doi: 10.1016/j.ijcard.2017.08.068

35. Masumoto A, Mohri M, Takeshita A. Three-year follow-up of the Japanese patients with microvascular angina attributable to coronary microvascular spasm. Int J Cardiol. 2001; 81(2-3):151-6. doi 10.1016/s0167-5273(01)00540-x

36. Crea F, Bairey Merz C, Beltrame J, Kaski J, Ogawa H, Ong P, et al. The parallel tales of microvascular angina and heart failure with preserved ejection fraction: a paradigm shift. Eur Heart J 2017; 38(7):473-477. doi: 10.1093/eurheartj/ehw461

37. Popovic B, Agrinier N, Bouchahda N, Pinelli S, Maigrat C, Metzdorf P, et al. Coronary embolism among ST-segment-elevation myocardial infarction patients: mechanisms and management. Circ Car- diovasc Interv. 2018; 11(1):e005587. doi: 10.1161/CIRCINTERVENTIONS.117.005587

38. Kleber F, Hauschild T, Schulz A, Winkelmann A, Bruch L. Epidemiology of myocardial infarction caused by presumed paradoxical embolism via a patent foramen ovale. Circ J. 2017; 81(10):1484-9. doi: 10.1253/circj.CJ-16-0995

39. Ghadri J, Wittstein I, Prasad A, Sharkey S, Dote K, Akashi Y, et al. International expert consensus document on Takotsubo syndrome (Part I): clinical characteristics, diagnostic criteria, and pathophysiology. Eur Heart J. 2018; 39(22):2032-46. doi: 10.1093/eurheartj/ehy076

40. Ghadri J, Wittstein I, Prasad A, Sharkey S, Dote K, Akashi Y, et al. International expert consensus document on Takotsubo syndrome (part II): diagnostic workup, outcome, and management. Eur Heart J. 2018; 39(22):2047-62. doi: 10.1093/eurheartj/ehy077

41. Pasupathy S, Tavella R, Beltrame J. The what, when, who, why, how and where of myocardial infarction with non-obstructive coronary arteries (MINOCA). Circ J. 2016; 80(1):11-6. doi: 10.1253/ circj.CJ-15-1096

42. Raparelli V, Elharram M, Shimony A, Eisenberg M, Cheema A, Pilote L. Myocardial infarction with no obstructive coronary artery disease: angiographic and clinical insights in patients with premature presentation. Can J Cardiol. 2018; 34(4):468-76. doi: 10.1016/j.cjca.2018.01.004

43. Lichtlen $\mathbf{P}$, Bargheer $\mathbf{K}$, Wenzlaff $\mathbf{P}$. Long-term prognosis of patients with anginalike chest pain and normal coronary angiographic findings. J Am Coll Cardiol. 1995; 25(5):1013-8. doi: 10.1016/07351097(94)00519-v

44. Nordenskjöld A, Baron T, Eggers K, Jernberg T, Lindahl B. Predictors of adverse outcome in patients with myocardial infarction with non-obstructive coronary artery (MINOCA) disease. Int J Cardiol. 2018; 261:18-23. doi: 10.1016/j.ijcard.2018.03.056

45. Smilowitz N, Mahajan A, Roe M, Hellkamp A, Chiswell K, Gulati M, et al. Mortality of myocardial infarction by sex, age, and obstructive coronary artery disease status in the action registry-gwtg (acute coronary treatment and intervention outcomes network registry-get with the guidelines). Circ Cardiovasc Qual Outcomes 2017; 10(12):e003443. doi: 10.1161/CIRCOUTCOMES.116.003443 